\title{
Assessment of the optic nerve, optic disc, and perineural area using shear-wave elastography in patients with multiple sclerosis
}

\author{
Suna Sahin Ediz ${ }^{1}$, Basak Atalay ${ }^{2}$, Ilknur Aydın Canturk², and Adnan Kabaalioglu ${ }^{3}$ \\ ${ }^{1}$ Istanbul Dr Lütfi Kırdar Kartal Eğitim ve Araştırma Hastanesi \\ ${ }^{2}$ Goztepe Egitim ve Arastirma Hastanesi \\ ${ }^{3}$ Koc University
}

January 18, 2021

\begin{abstract}
Purpose: To observe and describe the stiffness changes of the optic nerve in the patients with multiple sclerosis (MS) with or without optic neuritis and healthy adults via shear wave elastography (SWE). Methods: Seventy optic nerves from thirty-five patients with MS and sixty optic nerves from thirty healthy subjects were included prospectively in the study. The optic nerve $(\mathrm{ON})$, optic disc (OD), and perineural area were evaluated with SWE and optic nerve sheat diameter (ONSD) was measured by ultrasound. Results The mean age of patients was $39.68 \pm 9.99$ years. There was no statistically significant difference between the groups in terms of ONSD, SWE ON, SWE OD, and SWE perineural area levels (p>0.05). In the MS group; No statistically significant difference was found between patients with and without optic neuritis for the mean age, gender distribution, duration of MS, types of MS, ONSD, SWE ON, SWE OD, SWE perineural area, and Expanded Disability Status Scale (EDSS) scores ( $p>0.05$ ). No statistically significant difference in terms of ONSD, SWE ON, SWE OD, and SWE perineural area between the MS patients with or without optic neuritis and the control group ( $>$ > 0.05). Conclusion: Shear wave elastography measurements of the optic nerve, optic disc, and perineural area do not contribute to the evaluation of optic neuritis in a patient with MS.
\end{abstract}

Assessment of the optic nerve, optic disc, and perineural area using shear-wave elastography in patients with multiple sclerosis

Runnig Head:

"Shear-wave elastography findings of optic nerve in multiple sclerosis"

*Suna SAHIN EDIZ ${ }^{1}$, Basak ATALAY², Ilknur Aydin CANTURK³ ${ }^{3}$ Adnan KABAALIOGLU4

${ }^{1}$ Department of Radiology, University of Health Sciences, Kartal Dr. Lutfi Kirdar City Hospital, Istanbul, Turkey

${ }^{2}$ Department of Radiology, Medeniyet University, Goztepe Education and Research Hospital, Istanbul, Turkey

${ }^{3}$ Department of Neurology, Medeniyet University, Goztepe Education and Research Hospital, Istanbul, Turkey

${ }^{4}$ Department of Radiology, Koc University, Koc University Hospital, Istanbul, Turkey

\section{Corresponding Author}

Suna Sahin Ediz, MD

Department of Radiology 
University of Health Sciences, Kartal Dr. Lutfi Kirdar City Hospital, Istanbul, Turkey

Postal Address: Semsi Denizer St. E-5 Highway Cevizli Located Kartal/ISTANBUL

Postal Code: 34890

Phone: +905302270246

Fax: +902164583000

E-mail:suna-sahin@hotmail.com

ACKNOWLEDGEMENTS : We would like to thank our colleagues in the department of radiology and neurology.

\section{ABSTRACT}

Purpose: To observe and describe the stiffness changes of the optic nerve in the patients with multiple sclerosis (MS) with or without optic neuritis and healthy adults via shear wave elastography (SWE).

Methods : Seventy optic nerves from thirty-five patients with MS and sixty optic nerves from thirty healthy subjects were included prospectively in the study. The optic nerve (ON), optic disc (OD), and perineural area were evaluated with SWE and optic nerve sheat diameter (ONSD) was measured by ultrasound.

\section{Results}

The mean age of patients was $39.68 \pm 9.99$ years. There was no statistically significant difference between the groups in terms of ONSD, SWE ON, SWE OD, and SWE perineural area levels (p>0.05). In the MS group; No statistically significant difference was found between patients with and without optic neuritis for the mean age, gender distribution, duration of MS, types of MS, ONSD, SWE ON, SWE OD, SWE perineural area, and Expanded Disability Status Scale (EDSS) scores ( $p>0.05$ ). No statistically significant difference in terms of ONSD, SWE ON, SWE OD, and SWE perineural area between the MS patients with or without optic neuritis and the control group ( $\mathrm{p}>0.05)$.

Conclusion : Shear wave elastography measurements of the optic nerve, optic disc, and perineural area do not contribute to the evaluation of optic neuritis in a patient with MS.

Keywords : Multiple sclerosis; optic nerve; shear wave elastography; optic neuritis

\section{INTRODUCTION}

Multiple sclerosis is a chronic inflammatory disease and one of the most common causes of neurological disability in young adults globally. ${ }^{1,2}$ Many genetic and environmental factors, demyelination, and ironrelated abnormalities are the etiological and pathological causes of the MS. ${ }^{3-5}$

MS can affect all the anatomical parts of the visual system. The most often seen presentations are optic neuritis, brainstem, and spinal cord syndromes. ${ }^{1}$ Optic neuritis may be the first manifestation of multiple sclerosis. ${ }^{6}$ Acute demyelinating optic neuritis is the presenting symptom in about $20 \%$ of MS patients and affects about half of MS patients at some point in the disease course. ${ }^{7}$

Computerized tomography has limited applications in optic nerve pathologies because of the ionizing radiation and low resolution of soft tissue. Conventional and non-conventional Magnetic Resonance Imaging (MRI) such as diffusion tensor imaging, diffusion-weighted imaging provide high-resolution images. Whereas it is time-consuming, expensive, less obtainable, and often requires the use of gadolinium contrast. A more practical method is needed to evaluate the optic nerve for the initial diagnosis and follow-up of patients. ${ }^{8-10}$

Sonoelastography is an advanced sonographic technique and a non-invasive method for evaluating the different elasticity characteristics of tissues. There are two main sonoelastographic techniques that include strain elastography (SE), which display the local strain of a given sample of tissue (relative strain) and compresses the tissues axially, and shear wave elastography (SWE), which uses waves that are generated by transducers 
and interact with the tissue and a less operator-dependent method. Shear wave elastography can allow visualization and documentation of absolute stiffness in kilopascals or meters per second objectively without a maneuver. ${ }^{10,11}$

Sonoelastography has been used in the evaluation of internal organ pathology, and more recently, its clinical application to optic nerve tissue has been a growing area of interest. ${ }^{12}$ As far as we know, there is only one reported study about the sonoelastography of the optic nerve in MS patients in the English medical literature. ${ }^{13}$

The main purpose of the study is to assess the elasticity features of the optic nerve, optic disc, and perineuronal area using SWE in patients with MS (with or without optic neuritis), compare with healthy volunteers and describe the contribution of SWE findings for visual dysfunction in MS.

\section{MATERIAL AND METHODS}

\section{Participants}

This prospective study was approved by the local ethics committee and was conducted according to the Helsinki Declaration. All adult participants provided written informed consent to participate in the study.

Between February 2019 and August 2019, both optic nerves of patients with MS and healthy participants who were referred by the Neurology Clinic of our institution were examined. MS was diagnosed by an experienced neurologist in compliance with the revised McDonald criteria, and duration of the disease and the clinical rating scale called "Expanded Disability Status Scale (EDSS)" score was also recorded. Patients diagnosed with two types of MS; Relapsing-Remitting MS (RRMS) or Secondary-Progressive MS (SPMS) were included in the study. Patients had previous optic neuritis diagnosed based on typical history, clinical and radiological findings.

Patients with a history of orbital surgical intervention and trauma, systemic diseases (hypertension, diabetes mellitus), infections with optic nerve involvement (meningitis, syphilis, tuberculosis), disorders that cause optic nerve damage (cranial vasculitis, Graves' disease, autoimmune diseases, etc.) were excluded from the study.

\section{Equipment and scanning}

We used The Esaote QElaXto 2D sonography system (Esaote S.p.A., Genova, Italy) with SWE software sonoelastographic evaluations. A linear array probe of 6-9 $\mathrm{MHz}$ was used in the SWE examinations. The participants had a supine position with closed eyes during the examination. We applied the coupling gel between the eyelids of all the participants and instructed them not to move their eyes during the examination. We measured the optic nerve diameter (OND) for each eye between the external hypoechogenic borders of the optic nerve without hyperechogenic space surrounding that nerve by the B-mode US and then we applied SWE without compression. We standardized the examination technique based on literature. ${ }^{9,13,14}$ In addition to the optic nerve, we obtained the measurements of the perineural area and optic disc by SWE. We used the transverse plane from inside the equator of the eyeball and longitudinal axis of the optic nerve for measurements and then saved static images.

\section{Data analysis}

One radiologist performed sonoelastography measurements for all subjects. Two radiologists (S.S.E and A.K with 2 years of experience in elastography and 30 years of conventional US experience, respectively) determined the final interpretations through consensus. The radiologists were blind to the clinical information of the patients with MS (with or without optic neuritis).

Quantitatively analysis of optic nerve, optic disc, and perineural area stiffness was expressed as $\mathrm{kPa}$ with a color scale of $0-150 \mathrm{kPa}$ and a region of interest at an interval of 2 to $3 \mathrm{~mm}$. Three measurements for the quantitative value of each optic nerve, optic disc, and perineural area were acquired and the average values were used for the statistical analysis. (Fig1 and 2) 


\section{Statistical Analysis}

IBM SPSS Statistics 22 (IBM SPSS, Turkey) program was used in the analysis of the data obtained in the study. The suitability of the parameters to the normal distribution was evaluated by the Shapiro Wilks test. Besides descriptive statistical methods (mean, standard deviation, frequency), a student t-test was used for comparing normally distributed parameters between two groups, and the Mann Whitney U test was used for comparing non-normally distributed parameters between two groups while evaluating the study data. Pearson correlation analysis was used to examine the relationships between parameters that are suitable for normal distribution, and Spearman's rho correlation analysis was used to examine the relationships between parameters not compatible with a normal distribution. Continuity (yates) correction was used to compare qualitative data. Results were evaluated within the $95 \%$ confidence range at $\mathrm{p}<0.05$ significance level.

\section{RESULTS}

The study was conducted with 130 eyes of 65 patients, $44(67.7 \%)$ male and 21 (32.3\%) female, whose ages ranged from 18 to 59 . The mean age was $39.68 \pm 9.99$ years. The cases were divided into two groups as "MS Group" ( $\mathrm{n}=70)$ and "Control Group" $(\mathrm{n}=60)$. The duration of the disease in the MS group ranges between 2 and 25 years, with a mean of $14.74 \pm 5.85$ years and a median of 15 years. According to MS types; twenty-nine $(44.6 \%)$ of the cases had RRMS and six $(9.2 \%)$ had SPMS type. There was no statistically significant difference between the groups in terms of ONSD, SWE ON, SWE OD, and SWE perineural area levels $(\mathrm{p}>0.05)$. (Table 1$)$.

In the MS group; there is no statistically significant difference between patients with and without optic neuritis, in terms of mean age, gender distribution, MS duration, MS types, ONSD, SWE ON, SWE OD, SWE perineural area, and EDSS levels ( $\mathrm{p}>0.05)$. (Table 2). There is no statistically significant difference in terms of ONSD, SWE ON, SWE OD, and SWE perineural area between the MS patients with optic neuritis and the control group ( $\mathrm{p}>0.05)$. (Table 3 ). There is no statistically significant difference in terms of ONSD, SWE ON, SWE OD, and SWE perineural area between the MS patients without optic neuritis and the control group ( $\mathrm{p}>0.05)$. (Table 4)

DISCUSSION : MS is an autoimmune, chronic, inflammatory, and demyelinating disease. While MS can lead to optical neuritis, internuclear ophthalmoplegia, nystagmus, and saccadic dysmetria as visual dysfunction, optic neuritis is the most frequently detected one. ${ }^{15}$ It is often associated with severe visual loss. Therefore, early diagnosis of optic nerve damage is extremely important. Recently, ultrasonography has become an important adjuvant in the evaluation of the optic nerve. ${ }^{16}$ We performed this study considering that SWE can be an effective modality for evaluating the optic nerve in the early diagnosis of optic neuritis due to non-invasive and low-cost properties.

Volume loss and axonal damage in the retinal nerve fibers can be observed in MS patients. ${ }^{15}$ The optic disc usually appears normal but is mildly edematous in one-third of cases. ${ }^{17}$ Also, autoimmune-related posterior uveitis or vasculitis of the optic nerve in Wegener's granulomatosis may cause optic disc edema. ${ }^{18}$ This effect of autoimmune pathologies on the optic disc may cause damage by similar mechanisms in MS patients. The presence of the vascular network in the perineural area may play a role in the development of optical neuritis by having immune cells in this autoimmune inflammatory process. ${ }^{19}$ Thus, we thought that the evaluation of the perineural area might be beneficial and added this area to our research. In the literature, these parameters have been evaluated with MRI in different studies. ${ }^{20-22}$ However, there is no study in which all three parameters were evaluated together on SWE.

In the literature, there is only one study evaluating sonoelastographic findings in patients with MS, and only the optic nerve was evaluated in this study. ${ }^{13}$ Besides, in another study; Batur et al. evaluated both ON and retrobulbar tissue in patients with isolated optic neuritis on SWE and reported that comparison of the normal eye with isolated optic neuritis. The stiffness of the optic nerve in optic neuritis was found higher than the contralateral normal optic nerve and this difference was statistically significant. ${ }^{23}$ In our study, we aimed to develop a different point of view; the optic disc and perineural area were also evaluated in addition to the optic nerve, and quantitative evaluation was made particularly on SWE to increase the reliability of 
the study. Our study has been the first to evaluate all these parameters together. Besides, we strengthened the clinical effectiveness of our study by comparing our SWE measurements with the EDSS score. EDDS is widely used in clinical trials and for the assessment of MS. Since we thought the EDDS and sonoelastographic parameters might have a relation, we also compared the SWE results with the EDDS scores. Unfortunately, we could not find any significant differences.

In Inal et al.'s study, the sonoelastographic changes in the optic nerve in patients with MS were evaluated in terms of the elasticity of the optic nerve based on the three main colorings in strain elastography (SE) and SWE. ${ }^{13}$ According to this study, both SE and SWE can be used to investigate inflammation in patients with MS and may provide early diagnosis of optic neuritis. In our study, no statistically significant difference was found in the comparison of the optic nerve, optic disc, and perineural area SWE measurements between the MS patients (with or without optic neuritis attack) and healthy volunteers. According to these findings, our study results do not support the results which they reported.

At this point, the disadvantages of SWE arise such as the difficulty of the measurement technique and dependence on the operator. Optic nerve diameter up to $5 \mathrm{~mm}$ is considered normal in a healthy population. ${ }^{24,25}$ Inal et al. found that the mean optic nerve diameter was $5.31 \pm 0.48 \mathrm{~mm}$ (range, 3.8-6.5) in MS patients, while this value was $5.22 \pm 0.58 \mathrm{~mm}$ (range, $3.7-7$ ) in healthy volunteers. These findings support the concerns about the measurement. In our study, this value was $3.03 \pm 0.58 \mathrm{~mm}$ in the healthy population and it was compatible with the information in the literature.

Optic neuritis may cause increased or decreased ONSD in the acute and chronic phases. ${ }^{16,26,27}$ This may be an early and late sign of optic neuritis. The hypothesis that optic nerve thickness and stiffness may differ in optic neuritis with MS patients is the study's main purpose. However, we could not find any significant difference in ONSD and SWE ON between patient groups with and without control and optic neuritis. According to the optic neuritis stage, we anticipate that non-classified patients may have led to these results.

One of the limitations of our study is that SWE is an operator-dependent measurement method. Although our small size of the patient population seems to be a problem, the prospective study design increases the reliability of our results.

\section{CONCLUSION}

The measurements of SWE that we recommended as an easily accessible, non-invasive method that might be used in the evaluation of the optic nerve for visual dysfunction of patients with MS, do not contribute additionally.

CONFLICT OF INTEREST : There is no conflict of interest.

\section{REFERENCES}

1. Dobson R, Giovannoni G. Multiple sclerosis - a review. Eur J Neurol. 2019;26(1):27-40.

2. Oh J, Vidal-Jordana A, Montalban X. Multiple sclerosis: clinical aspects. Curr Opin Neurol. 2018;31(6):752-759.

3. Ascherio A. Environmental factors in multiple sclerosis. Expert Rev Neurother. 2013;13(12 Suppl):3-9.

4. Assaf Y, Chapman J, Ben-Bashat D, et al. White matter changes in multiple sclerosis: correlation of q-space diffusion MRI and 1H MRS.Magn Reson Imaging. 2005;23(6):703-710.

5. Chawla S, Kister I, Wuerfel J, et al. Iron and Non-Iron-Related Characteristics of Multiple Sclerosis and Neuromyelitis Optica Lesions at 7T MRI. AJNR Am J Neuroradiol. 2016;37(7):1223-1230.

6. Wilhelm H, Schabet M. The Diagnosis and Treatment of Optic Neuritis.Dtsch Arztebl Int. 2015;112(37):616-625; quiz 626.

7. Balcer LJ. Clinical practice. Optic neuritis. N Engl J Med.2006;354(12):1273-1280. 
8. Ricci P, Maggini E, Mancuso E, Lodise P, Cantisani V, Catalano C. Clinical application of breast elastography: state of the art. Eur J Radiol. 2014;83(3):429-437.

9. Vural M, Acar D, Toprak U, et al. The evaluation of the retrobulbar orbital fat tissue and optic nerve with strain ratio elastography.Med Ultrason. 2015;17(1):45-48.

10. Yang YP, Xu XH, Guo LH, et al. Qualitative and quantitative analysis with a novel shear wave speed imaging for differential diagnosis of breast lesions. Sci Rep. 2017;7:40964.

11. Ogurel T, Burulday V. Strain and Shear Wave Elastography in Diagnosis of Retrobulbar Neuritis. $J$ Neuroophthalmol.2020;40(2):169-173.

12. Ophir J, Cespedes I, Garra B, Ponnekanti H, Huang Y, Maklad N. Elastography: ultrasonic imaging of tissue strain and elastic modulus in vivo. European journal of ultrasound. 1996;3(1):49-70.

13. Inal M, Tan S, Yumusak EM, Sahan MH, Alpua M, Ornek K. Evaluation of the optic nerve using strain and shear wave elastography in patients with multiple sclerosis and healthy subjects. Med Ultrason.2017;19(1):39-44.

14. Sahan MH, Dogan A, Inal M, Alpua M, Asal N. Evaluation of the Optic Nerve by Strain and Shear Wave Elastography in Patients With Migraine.J Ultrasound Med. 2019;38(5):1153-1161.

15. Hoff JM, Dhayalan M, Midelfart A, Tharaldsen AR, Bo L. Visual dysfunction in multiple sclerosis. Tidsskr Nor Laegeforen.2019;139(11).

16. Perez Sanchez S, Eichau Madueno S, Rus Hidalgo M, et al. Usefulness of optic nerve ultrasound to predict clinical progression in multiple sclerosis. Neurologia. 2018.

17. Beck RW, Cleary PA, Anderson MM, Jr., et al. A randomized, controlled trial of corticosteroids in the treatment of acute optic neuritis. The Optic Neuritis Study Group. N Engl J Med.1992;326(9):581-588.

18. Chan JW. Optic neuritis in multiple sclerosis. Ocul Immunol Inflamm. 2002;10(3):161-186.

19. Green AJ, McQuaid S, Hauser SL, Allen IV, Lyness R. Ocular pathology in multiple sclerosis: retinal atrophy and inflammation irrespective of disease duration. Brain. 2010;133(Pt 6):1591-1601.

20. Soderstrom M, Ya-Ping J, Hillert J, Link H. Optic neuritis: prognosis for multiple sclerosis from MRI, CSF, and HLA findings.Neurology. 1998;50(3):708-714.

21. Swanton JK, Fernando KT, Dalton CM, et al. Early MRI in optic neuritis: the risk for clinically definite multiple sclerosis. Mult Scler. 2010;16(2):156-165.

22. Cellina M, Floridi C, Rosti C, et al. MRI of acute optic neuritis (ON) at the first episode: Can we predict the visual outcome and the development of multiple sclerosis (MS)? Radiol Med.2019;124(12):1296-1303.

23. Batur M, Batur A, Cilingir V, et al. Ultrasonic Elastography Evaluation in Optic Neuritis. Semin Ophthalmol.2018;33(2):237-241.

24. Kim DH, Jun JS, Kim R. Measurement of the Optic Nerve Sheath Diameter with Magnetic Resonance Imaging and Its Association with Eyeball Diameter in Healthy Adults. J Clin Neurol.2018;14(3):345-350.

25. Vaiman M, Abuita R, Bekerman I. Optic nerve sheath diameters in healthy adults measured by computer tomography. Int J Ophthalmol.2015;8(6):1240-1244.

26. De Bernardo M, Vitiello L, Rosa N. Optic nerve ultrasound measurement in multiple sclerosis. Acta Neurol Scand.2019;139(4):399-400.

27. Kwon YJ, Kim YH, Baek SH, Son MH, Lee JH, Kim BJ. Transorbital ultrasonography in acute optic neuritis: Can it be a supportive diagnostic tool? Mult Scler Relat Disord. 2019;31:54-58.

Table 1: The detailed SWE findings of both groups are described. 


\begin{tabular}{llll}
\hline & MS $(\mathbf{n}=\mathbf{7 0})$ & Control $(\mathbf{n}=\mathbf{6 0})$ & $\mathbf{p}$ \\
\hline ONSD Mean \pm SD $_{\text {SWE ON Median (IQR) }}$ & $2,94 \pm 0,50$ & $3,03 \pm 0,58$ & ${ }^{1} 0,319$ \\
SWE OD Median (IQR) & $7,31(18,5)$ & $7,30(6,1)$ & ${ }^{2} 0,957$ \\
SWE Perineural Area Median (IQR) & $8,07(15,6)$ & $7,28(9,2)$ & ${ }^{2} 0,724$ \\
\hline
\end{tabular}

${ }^{1}$ Student $t$ test ${ }^{2}$ Mann Whitney U Test

Table 2: The detailed findings associated with or without optic neuritis such as duration of MS, type of MS, and SWE findings on the MS group are described.

\begin{tabular}{llll}
\hline & ON & ON & $\mathbf{p}$ \\
\hline & Absence $(\mathbf{n = 4 5 )}$ & Presence $(\mathbf{n = 2 5})$ & \\
Duration of MS Median (IQR) & $12(9,5)$ & $17(6)$ & ${ }^{3} 0,243$ \\
ONSD Mean \pm SD & $2,87 \pm 0,52$ & $3,06 \pm 0,43$ & ${ }^{1} 0,119$ \\
SWE ON Median (IQR) & $7,64(12,7)$ & $6,3(24,5)$ & ${ }^{3} 0,783$ \\
SWE OD Median (IQR) & $8,97(16,0)$ & $5,45(11,1)$ & ${ }^{3} 0,157$ \\
SWE Perineural Area Median (IQR) & $8,44(12,4)$ & $13,47(18,4)$ & ${ }^{3} 0,433$ \\
EDSS Median (IQR) & $2(0,5)$ & $2(1)$ & ${ }^{3} 0,979$ \\
Type of MS n(\%) & & & \\
RRMS & $36(\% 80)$ & $22(\% 88)$ & ${ }^{4} 0,517$ \\
SPMS & $9(\% 20)$ & $3(\% 12)$ & \\
\hline
\end{tabular}

${ }^{1}$ Student t-test ${ }^{2}$ Continuity (yates) düzeltmesi ${ }^{3}$ Mann Whitney U Test ${ }^{4}$ Fisher's Exact Test

Table 3: The comparison of SWE values between MS patients with optic neuritis and control group

\begin{tabular}{llll}
\hline & ON Presence $(\mathbf{n}=\mathbf{2 5})$ & Control $(\mathbf{n}=\mathbf{6 0})$ & $\mathbf{p}$ \\
\hline ONSD Mean \pm SD $_{\text {SWE ON }}$ Median $(\mathrm{IQR})$ & $3,06 \pm 0,43$ & $3,03 \pm 0,58$ & ${ }^{1} 0,819$ \\
SWE OD Median (IQR) $_{\text {SWE Perineural Area Median }(\mathrm{IQR})}$ & $6,3(24,5)$ & $7,30(6,1)$ & ${ }^{2} 0,981$ \\
& $13,47(18,4)$ & $7,28(9,2)$ & ${ }^{2} 0,101$ \\
\hline
\end{tabular}

${ }^{1}$ Student $t$ test ${ }^{2}$ Mann Whitney $U$ Test ${ }^{*} p<0.05$

Table 4: The comparison of SWE values between MS patients without optic neuritis and control group

\begin{tabular}{llll}
\hline & ON Absence $(\mathbf{n = 4 5 )}$ & Control $(\mathbf{n}=\mathbf{6 0})$ & $\mathbf{p}$ \\
\hline ONSD Mean \pm SD $_{\text {SWE ON Median (IQR) }}$ & $2,87 \pm 0,52$ & $3,03 \pm 0,58$ & ${ }^{1} 0,139$ \\
SWE OD Median (IQR) $_{\text {SWE Perineural Area Median (IQR) }}$ & $8,64(12,7)$ & $7,30(6,1)$ & ${ }^{2} 0,954$ \\
\hline
\end{tabular}


${ }^{1}$ Student $t$ test ${ }^{2}$ Mann Whitney $U$ Test ${ }^{*} p<0.05$

FIGURE 1: Grayscale (left) and color (right) SWE images of the optic nerve and circular regions of interest (box). Elasticity measurement with SWE of the optic nerve of a 38-year-old female patient with MS revealed a mean score of $22.55 \mathrm{kPa}$.

FIGURE 2: Grey-scale (left) and color (right) SWE images showing the measurement technique a) on the perineural area and $\mathrm{b}$ ) on the optic disc.

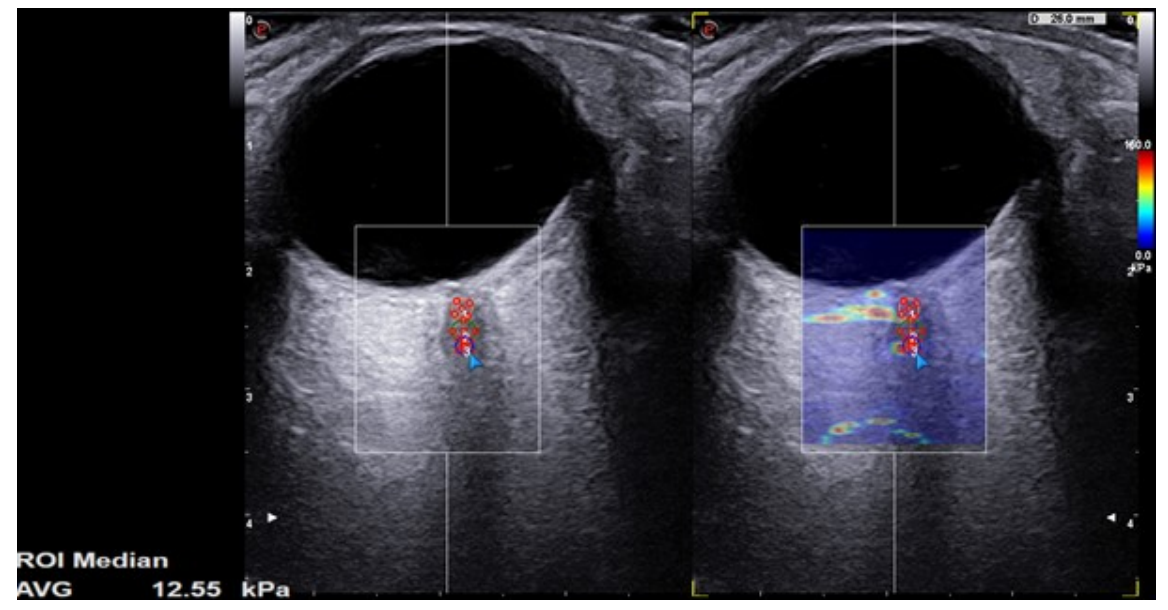




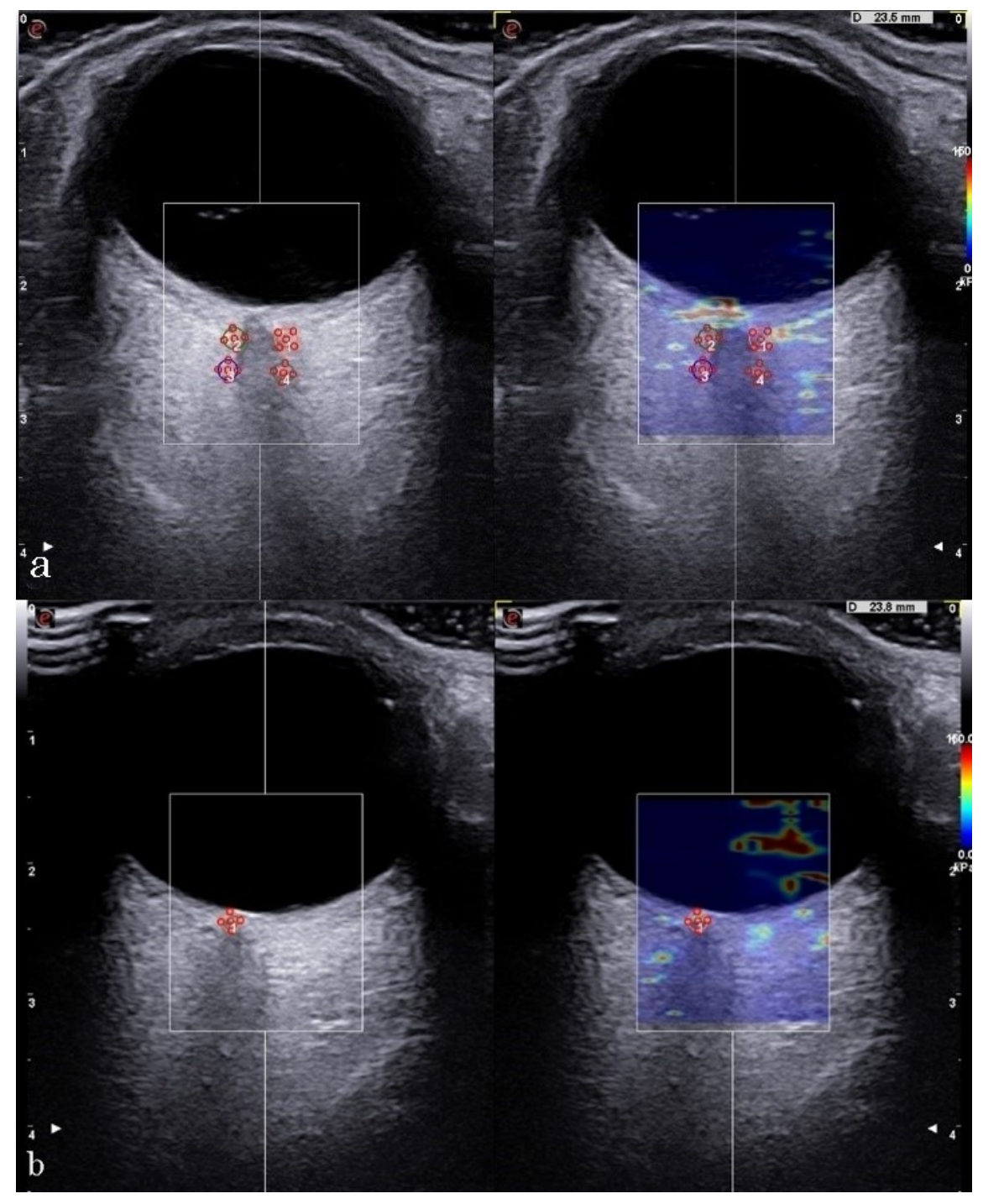

